\title{
Identification of Bacteria from Skin Swab in Pre-operative Closed Fracture Orthopedic Surgery Patients at Dr. Hasan Sadikin General Hospital
}

\author{
Ghitaa Bengtissen, ${ }^{1}$ Sunarjati Sudigdoadi, ${ }^{2}$ Yoyos Dias Ismiarto, ${ }^{3}$ Isa Ridwan ${ }^{3}$ \\ ${ }^{1}$ Faculty of Medicine Universitas Padjadjaran, Indonesia, ${ }^{2}$ Department of Biomedical Sciences \\ Faculty of Medicine Universitas Padjadjaran, Indonesia, ${ }^{3}$ Department of Orthopedic and \\ Traumatology Faculty of Medicine Universitas Padjadjaran/Dr. Hasan Sadikin General Hospital \\ Bandung, Indonesia
}

\section{Abstract}

Background: Surgical site infection (SSI) is the most frequent site of health care- associated infections (HAIs). Surgical incision breaks the protective barrier of the skin and causes bacteria to enter; therefore, pre-operative procedure is very important to reduce the risk of SSI. This study aimed to identify any bacteria in skin swab of pre-operative closed fracture during elective orthopedic surgery patients.

Methods: This was a descriptive laboratory study, conducted in the Department of Microbiology, Faculty of Medicine, Universitas Padjadjaran in October to November 2012. Samples were taken from skin swab of patients with closed fracture taken before elective orthopedic surgery. Samples were cultured on blood agar, then incubated in an aerobic condition for 24 hours at $37^{\circ} \mathrm{C}$. The bacteria were then identified, including the type and the number of the bacteria colonies, using microscopic gram staining and by biochemistry testing using coagulase, novobiocin, and catalase test. The bacteria colonies formed on blood agar were counted using CFU/mL.

Results: Of 24 samples taken, 14 (58.3\%) were positive for bacteria, 7 negative and 3 were excluded due to dead bacteria. The bacteria identified in the samples were all staphylococcus species and the colony counting was lower than $10^{5} \mathrm{CFU} / \mathrm{mL}$.

Conclusions: Bacteria staphylococcus are detected from more than half of skin swab during preoperative closed fracture orthopedic surgery patients. Thus, it is important to apply a proper antiseptic procedure before making a surgical incision to reduce the risk of SSI.

Keywords: Bacteria, pre-operative patient's skin, Staphylococcus

\section{Introduction}

According to the Centers for Disease Control and Prevention (CDC), the surgical site of infection (SSI) is defined as a proliferation of pathogenic microorganism that develops in an incision site. ${ }^{1}$ Surgical site infections are known as the third most frequent site of health care-associated infections (HAIs). ${ }^{2}$

Based on surveysin five hospitals in the UK, the National Nosocomial Infection Surveillance (NNIS) system reported aprevalence of hospital acquired-infection (HAI) relating to surgical wounds as high as $10 \%$. Moreover, a prevalence survey that has been conducted suggested that at least $5 \%$ of patients who had undergone a surgical procedure were found to have developed SSI. In the other hand, SSI accounts for $14 \%$ to $16 \%$ of the estimated 2 million nosocomial infections affecting hospitalized patients in the USA. ${ }^{1,3,4}$

For surgical site infections, the initial exposure of microbial pathogens occurs most often during the surgical procedure that performed in the operating theatre (OT), therefore, we should be aware of the risk of surgical site infections that is influenced by the characteristics of the patient, operation, and health care facility. ${ }^{2}$

The normal flora of the patient's skin is 
the source of pathogens for most surgical site infections. When the physician open or make skin incision, the tissue that is exposed will be at risk for contamination with normal flora. ${ }^{2}$ The skin always harbors a variety of microorganisms that can be divided into two groups; the resident flora and transient flora. ${ }^{5}$ The superficial layers of the skin are colonized by transient flora; while the deep layer of the skin are attached by the normal flora resident such as Staphylococcus epidermidis. ${ }^{6}$ Other normal bacterial flora in the skin are among others Staphylococcus aureus, Staphylococcus saprophyticus, diphtheroids, Micrococcus species and asmall number of other organisms such as Candida species, Acinetobacter species, and many others. ${ }^{5}$ Under certain condition, this normal flora may become pathogenic as they enter the blood stream resulting in disease. ${ }^{5}$

Surgical wounds are classified by the degree of bacterial contamination or microbial loads during the time of the procedure. The greater the microbial loads, the higher the risk of infection. The Centers for Disease Control and Prevention (CDC), classifies wounds as clean, clean-contaminated, contaminated and dirty wounds. ${ }^{7}$ Orthopedic surgery as general surgery can be divided into 2 groups, elective surgery and emergency surgery. Elective surgery is the surgery that is planned in advanced and can safely be delayed for more than 24 hours while emergency surgery is the type of surgery that need to be performed in response to an urgent medical need or lifethreatening within 24 hours. ${ }^{8,9}$ Fracture is defined as a break in the bone, and classified into 2 types; open and closed fracture. Open fracture is defined as broken bone that penetrates the skin; whereas closed fracture is a broken bone that does not penetrate the skin. ${ }^{10}$ According to CDC criteria, a surgical site that is contaminated with $>10^{5}$ microorganisms per gram of tissue, has been shown to have an increased risk of SSI. Nevertheless, the contaminating microorganisms concentration is required to cause infection and that is much lower when foreign material is present at the surgical site; for example, 100 staphylococci per gram tissue. ${ }^{2,7,11}$ Thus, pre-operative patients' skin hygiene is very crucial prior asurgery to prevent post-operative complications. It is not only the responsibility of the physicians to make sure of this, as the patients themselves should take measures to avoid any postoperative infections. Therefore, skin care is imperative for pre-operative patients. ${ }^{12}$

The CDC recommends a patient to take a shower with an antiseptic solution or with a normal bath soap and warm water the night before surgery. This skin microbial colony counts will decrease, but it has not been shown definitively to reduce SSI rates. The CDC notes that the most commonly used skin antiseptic is chlorhexidinegluconate (CHG). ${ }^{13-15}$

Another way to prevent infection is by applying antiseptics to the skin of pre-operative patients. The role of an antiseptic is to inhibit the growth or action of microorganisms. It is used for skin before a clinical procedure. The United States Food and Drug Administration (FDA) has defined that an antiseptic is an agent that is able to decrease the number of bacteria on the skin. Antiseptics that are suggested by CDC for skin preparation are alcohol, chlorhexidinegluconate, and iodine/ iodophors. ${ }^{16}$

The aim of this study was to identify the presence of bacteria in skin swab, including the type of bacteria and the number of bacteria coloniesformed on blood agar.

\section{Methods}

The study method was descriptive laboratory study using skin swabs from previous study. In brief, skin swabs from patients in Dr. Hasan Sadikin Hospital in Bandung were taken, before an elective orthopedic surgery of closed fracture during the period of February to April 2012. ${ }^{17}$ The skin swab was taken 30-60 minutes before applying the antiseptic, and stored until used in this current study. Sample selection was performed using consecutive sampling method. The inclusion criteria in the previous study was skin swab from preoperative closed fracture orthopedic patients before applying antiseptic. The exclusion criteria were skin swab from pre-operative closed fracture orthopedic surgery patientthat was already infected, or that was cleaned with an antiseptic soap. The identification of the bacteria in this current study was conducted in October-November 2012, thus, bacteria in asample that died due to a longer time of incubation was excluded.

In this current study, the swab was cultured using Levine technique. The swab collection was placed in a sterile tube containing $1 \mathrm{~mL}$ of brain heart infusion (BHI) broth as a transport media, thenspread over the entire blood agar plate, prior incubation in an aerobic condition at $37^{\circ} \mathrm{C}$ for 24 hours. Any presence of bacteria on blood agar was identified, including the type of bacteria and the number of bacteria colonies. Colonies from blood agar were taken 
Table 1 The Presence of The Bacteria in A Closed Fracture Skin Swab on Blood Agar during A Pre-operative Elective Orthopedic Surgery

\begin{tabular}{lcc}
\hline \multicolumn{1}{c}{ Presence of bacteria } & Sample & Percentage (\%) \\
\hline Positive & 14 & 58.3 \\
Negative & 7 & 29.2 \\
Excluded* & 3 & 12.5 \\
\hline
\end{tabular}

Note: * Excluded due to dead bacteria on blood agar

for gram staining to identify Gram-positive or Gram-negative bacteria. Gram-positive bacteria retained a purple color of the crystal violet in its cell wall, whereas Gram-negative bacteria were stained red with safranin.

To differentiate the staphylococcus from streptococcus, catalase test was performed resulting in a positive or negative result, respectively. In brief, one drop of 3\% hydrogen peroxide was brought on a slide using a pipette. A colony from blood agar was taken, and the formation of bubbles was observed, indicating the production of oxygen and the catalase test was thus positive. ${ }^{18}$

To differentiate Staphylococcus aureus from other coagulase-negative staphylococci, coagulase test was performed. Two test tubes labeled as 'control' and 'test' was prepared, filled in with $1 \mathrm{~mL}$ of 1 in 10 diluted citrated plasma. Sterile broth and sample culture of $0.2 \mathrm{~mL}$ each was put into the test tubes, prior further incubation at $37^{\circ} \mathrm{C}$ for four hours. Coagulase test was positive when gelling of the plasma remained in place after inverting the tube, indicating S.aureus; whereas coagulase test was negative, indicating S.epidermidis and S.saprophyticus. ${ }^{18}$

Novobiocin test was performed to assess the susceptibility of bacteria to antibiotic. An antibiotic-impregnated disk containing novobiocinwas placed in the center of the agar, prior incubation at $35-37^{\circ} \mathrm{C}$ for 24 hours, and the bacteria growth on the surface of the plate was observed. A visible zone surrounding the disk was the zone of inhibition, indicating thesusceptibility of bacteria to the antibiotic. When there was no zone present or zone of inhibition was $<16$ $\mathrm{mm}$, the bacteria was resistant to the antibiotic such as $S$. saprophyticus. Zone of inhibition $>16$ $\mathrm{mm}$ indicated that bacteria was sensitive to novobiocin such as S. epidermidis. ${ }^{19}$

\section{Results}

This study was to identify the presence of the bacteria in skin swab, including the type of bacteria and the number of bacteria coloniesformed on blood agar. After 24-hour period of time, the presence of bacteria on the blood agar were identified. Of 24 samples taken, $14(58.3 \%)$ were positive for bacteria, 7 negative and 3 were excluded due to dead bacteria (Table 1).

These samples were then viewed under a microscope to identify the type of bacteria. Blood agar that contained more than one colony was isolated twice to explore whether there was possibly more than one type of bacteria. Of those 14 positive samples, 12 samples had one colony and 2 samples had two colonies, and the number of bacteria colonies were expressed in colony-forming units (CFU) per $\mathrm{mL}$, ranging from $1.0 \times 102$ to $1.87 \times 104$ $\mathrm{CFU} / \mathrm{mL}$ (Table 2). The type of bacteria present in the skin swab was Staphylococcus aureus

Table 2 The Number of Bacteria Colonies

\begin{tabular}{cc}
\hline Sample & $\begin{array}{c}\text { Colony Forming Units } \\
\text { (CFU/mL) }\end{array}$ \\
\hline 1 & $6.0 \times 10^{2}$ \\
2 & $1.13 \times 10^{4}$ \\
3 & $1.87 \times 104$ \\
4 & $2.1 \times 103$ \\
5 & $1.0 \times 10^{3}$ \\
6 & $1.0 \times 10^{2}$ \\
7 & $3.0 \times 10^{2}$ \\
8 & $2.0 \times 10^{2}$ \\
9 & $3.0 \times 10^{2}$ \\
10 & $2.0 \times 10^{2}$ \\
11 & $5.0 \times 10^{2}$ \\
12 & $1.1 \times 10^{3}$ \\
13 & $2.0 \times 10^{2}$ \\
14 & $1.0 \times 10^{2}$ \\
\hline Note: CFU= colony forming units $(\mathrm{CFU})$
\end{tabular}


$(\mathrm{n}=5)$, Staphylococcus epidermidis $(\mathrm{n}=7)$, and Staphylococcus saprophyticus $(\mathrm{n}=4)$.

\section{Discussion}

In this study, the type of surgery that has been performed on all patients is an elective surgery for closed fracture, that is a planned surgery. Therefore, it is categorized as clean surgery because the wound is clean and there is no acute inflammation that has been encountered. ${ }^{7}$ However, there are various bacteria growingon blood agar from the skin swab of pre-operative closed fracture elective orthopedic surgery patients; consisting of Staphylococcus species such as $S$. aureus, $S$. epidermidis, and S. saprophyticus. According to the National Nosocomial Infections Surveillance System (NNIS), the pathogens that most commonly isolated from surgical site infections (SSI) are S. aureus (20\%) and coagulase-negative staphylococci such as $S$. epidermidis and S. saprophyticus $(14 \%) .{ }^{13}$ Our study result shows that that the mostly found bacteria is $S$. epidermidis compared to $S$. aureus and S. saprophyticus.

Staphylococcus species are acquired from the patient's own skin flora. Intact skin acts as a very effective barrier and most infections cannot pass through intact skin. A surgical incision will create a break in that protective barrier, causing the bacteria to enter the bloodstream and resulting in diseases. Usually, normal flora of the patient's skinis the source of pathogens for most surgical site infections when the incision is performed. This is because of changing in the environment of normal flora. The precursor of surgical site infection (SSI) is the microbial contamination during a surgical procedure. According to CDC criteria, a surgical site which is contaminated with $>10^{5}$ microorganisms per gram of tissue has been shown to have an increased risk of SSI. Thus, the greater the microbial loads, the higher the risk of infection. ${ }^{2,7,11}$ However, there is no risk of infection because the number of colonies of bacteria is not more than $10^{5} .^{17}$ This is because all patients that were selected in this study were advised to take a shower with a normal bath soap at least the night before or in the morning of the operative day before they enter the operating room. A preoperative shower can decrease the count of skin microbial colonies but it has not been shown to ease SSI rates.

Bacteria from the skin can be divided into two categories, namely transient and resident flora. Transient flora colonizes the superficial layers of the skin while resident flora attached to deeper layers of the skin. An example oftransient flora is $S$. aureus, while resident flora is coagulase negative Staphylococci such as $S$. epidermidis and S. saprophyticus. ${ }^{6}$ In this study, the bacteria that mostly found was $S$. epidermidis compared to $S$. aureus. This is because transient flora iseasier to remove compared to resident flora that ismore resistant to removal.

Even though there is no risk of infections, patients should be aware of their own skin hygiene before they undergo surgery to prevent complications of post-operative. This is because there are other factors that can cause SSI such as the general state of the patient, intraoperative factors like the operating room environment (ventilation and cleanliness), sterilization of instrument, surgical attire like masks, caps, glove and gowns, and postoperative factors like length of stay in hospital after the surgery. ${ }^{11}$ Antiseptic is thus very crucial for pre-operative patients to decrease the risk of surgical site infection, as it can inhibit the growth or action of microorganisms.

In conclusion, there is a presence of bacteria from skin swab of pre-operative closed fracture elective orthopedic surgery patients, such as staphylococcus species like $S$. aureus, S. epidermidis and S. saprophyticus with acceptable colony forming units. Therefore, it is important to apply a proper antiseptic procedure before making a surgical incision to reduce the risk of SSI.

\section{References}

1. Bagnall NM, Vig S, Trivedi P. Surgicalsite infection. Surgery (Oxford). 2009;27(10):426-30.

2. Mangram AJ, Horan TC, Pearson ML, Silver LC, Jarvis WR. Guideline for prevention of surgical site infection 1999. Center for Disease Control and Prevention (CDC) Hospital Infection Control Practices Advisory Committee. Am J Infect Control. 1999;27(2):97-132.

3. Zinn J, Jenkins JB, Swofford V, Harrelson B, McCarter S. Intraoperative patient skin preparation agents: is there a difference ? AORN J. 2010;92(6):662-74.

4. Afifi IK, Baghagho EA. Three months study of orthopaedic surgical site infections in an Egyptian University hospital. Int J Infect Control. 2010;6(1):1-6.

5. Brooks GF, Carroll KC, Butel JS, Morse SA. Jawetz, Melnick, \& Adelberg's Medical Microbiology. 24 ${ }^{\text {th }}$ ed. Michigan US: Mc 
Graw Hill; 2004.

6. Humphreys H, Cunney R, Devitt E, Crowe M, Hayes B, O'Connor M, et al. Guidelines for hand hygiene in Irish healthcare settings. Ireland: SARI by HSE, Health Protection Surveillance Centre; 2004.

7. Greene LR. Guide to the elimination of orthopedic surgery surgical site infections: an executive summary of the Association for Professionals in Infection Control and Epidemiology elimination guide. Am J Infect Control. 2012:40(4);384-6.

8. New South Wales Nurses Association. Expert panel review of elective surgery and emergency access targets under the national partnership agreement on improving public hospital. The Lamp [Online Journal] 2012; Oct 1 [cited 2013 January 23]. Available from: https://www. thefreelibrary.com

9. Royal Australasian College of Surgeons. The case for the separation of elective and emergency surgery. Melbourne: Royal Australasian College of Surgeons; 2011. [cited 2013 January 23]. Available from: http://www.surgeons.org/ media/307115/sbm_2011-05-24 _ separating_elective_and_emergency_ surgery.pdf.

10. Ortholnfo. Diseases \& condition: open fractures. American Academy of The Orthopedic Surgeons; 2012 [cited 2012 April 2]; Available from: http://orthoinfo. aaos.org/topic.cfm?topic $=$ A00582

11. World Health Organization. WHO guidelines for safe surgery 2009: safe surgery saves lives. Geneva: WHO Press; 2009 [cited 2012 April 20]; Available from: https://apps.who.int/iris/bitstream/ handle/10665/44185/9789241598552 eng.pdf;jsessionid $=25$ ED3F5B06FE8F3C 0 E1D8156911CC48F?sequence $=1$
12. Johnson J. Pre-operative hygiene. HygieneExpert [Online] 2010 [cited 2012 April 3]. Available from: http:// www.hygieneexpert.co.uk/preoperativehygiene.html.

13. Seal LA, Paul-Cheadle D. A systems approach to preoperative surgical patient skin preparation. Am J Infect Control. 2004;32(2):57-62.

14. Rauk PN. Educational intervention, revised instrument sterilization methods, and comprehensive preoperative skin preparation protocol reduce cesarean section surgical site infections. Am J Infect Control. 2010;38(4):319-23.

15. Napolitano LM. Decolonization of the skin of the patient and surgeon. Surgical infections. Surgical Infections. 2006;7(Suppl 3):S3-15.

16. Parks PJ. Patient preoperative skin preparations to reduce surgical site infections. Future Direction in Surgery. 2006;1:84-7

17. RidwanI, Ismono D, IsmiartoYD, Hidajat NN. Comparison of effectiveness in decreasing bacterial counts between povidone iodine $10 \%$ painted with and without alcohol $70 \%$ wiping off preoperative skin preparation in orthopaedic surgery patients [Thesis]. Bandung: Universitas Padjadjaran; 2012.

18. Virtual Amrita Laboratories Universalizing Education. Catalase and coagulase test. Amrita Vishwa Vidyapeetham [Online] 2011 [cited 2012 April 15]. Available from: http://amrita.vlab.co.in/?sub=3\&br $\mathrm{ch}=73 \& \operatorname{sim}=703 \& \mathrm{cnt}=2$.

19. Hardy Diagnostics. Novobiocin differentiation disks. Santa Maria: Hardy Diagnostic [Online Article] 1996 [cited 2012 April 15]. Available from : https:// catalog.hardydiagnostics.com/cp_prod/ content/hugo/NovobiocinDiffDisks.htm 